\title{
Post-Intensive Care Syndrome
}

\author{
Marcela Káňová ${ }^{1,2 *}$ and Jan Máca ${ }^{1,2}$ \\ ${ }^{1}$ Department of Anesthesiology and Intensive Care Medicine, University Hospital, Ostrava, Czech Republic \\ ${ }^{2}$ Institute of Physiology and Pathophysiology, Faculty of Medicine, University of Ostrava, Czech Republic
}

*Corresponding author: Marcela Káňová, Department of Anesthesiology and Intensive Care Medicine, Institute of Physiology and Pathophysiology, Faculty of Medicine, University Hospital, Ostrava, Czech Republic

To Cite This Article: Marcela Káňová, Jan Máca, Post-Intensive Care Syndrome. 2020 - 10(6). AJBSR.MS.ID.001577.

DOI: 10.34297/AJBSR.2020.10.001577.

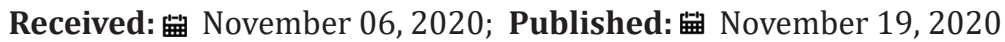

\begin{abstract}
Introduction
The world currently deals with the SARS-CoV-2 pandemic and its clinical form of Coronavirus Disease, first described in 2019 (COVID-19). Millions of patients suffer from dyspnea and low oxygenation, and many require artificial mechanical ventilation for acute respiratory failure. The most prominent problem is Acute Respiratory Distress Syndrome (ARDS), a life-threatening gas exchange impairment resulting in hypoxemia, hypercapnia followed by critical tissue hypoxia. The best ventilatory setting in ARDS, reducing the risk of Ventilator-Induced Lung Injury (VILI) is still a matter of debate in COVID-19. Other issues are the difficulties of diagnosing and treating the microbial super-infection, septic shock, and Multiple Organ Dysfunction (MODS). Intensive care medicine enhanced during the last decades resulting in the short-term outcomes (28day survival) improvement. However, the long-term outcomes, i.e., survivors' quality of life, stay the same. In part, it is due to the growing amount of elderly critically ill patients. Age together with obesity and other chronic diseases (metabolic syndrome, type 2 diabetes mellitus, cardiovascular diseases, liver and kidney diseases, sarcopenia, autoimmune disorders, cancer, etc.), are the main risk factors for an inappropriate inflammatory response to viral infection, e g, caused by SARS-CoV-2 [1].
\end{abstract}

Keywords: Microbial, CoVID-19, Diabetes mellitus, Critical tissue hypoxia, Life-threatening gas, Multiple Organ Dysfunction, Metabolic syndrome, Cardiovascular diseases, Ventilatory, Sarcopenia, Mitochondrial dysfunction, Biological

\section{The Inflammaging}

The inflammation, an age-related chronic subclinical systemic inflammation, might be the cause of more significant long-term consequences in older people. Acute viral infection sets in motion some biological processes such as genomic instability, oxidative stress, mitochondrial dysfunction, metabolism impairment. The acute cytokine storm is followed by a counter-regulatory antiinflammatory reaction, immunosuppression with increased susceptibility to the microbial super-infection. These alterations may trigger Post-Intensive Care Syndrome (PICS) followed by prolonged illness suffering and poor long-term prognosis [2].

\section{PICS}

The number of critical illness survivors is growing, but most of them suffer from PICS. The syndrome manifests in three areas: cognitive, physical, and mental impairment, and lasts long. Some dysfunctions persist from months to years [3].

\section{Cognitive impairment}

Cognitive disorder consists of impaired memory, attention, language, and visual-spatial abilities. There is strong evidence that critically ill patients undergoing delirium are at a greater risk of cognitive dysfunction, including dementia. Delirium is a common problem in the Intensive Care Unit (ICU) setting. The onset of delirium is frequently present in the case of more severe illness or when complications develop. The mainstay of delirium management is early detection using the screening tools such as the Confusion Assessment Method for the ICU (CAM-ICU) and care bundle [4].

\section{Physical impairments}

The physical impairments often develop due to acute illness catabolism followed by protein breakdown, loss of muscle mass and strength, and immobility. Weakness, muscle atrophy, Critical 
Illness Polyneuropathy (CIP), and Critical Illness Myopathy (CIM) are common complications of critically ill patients. Up to $80 \%$ of ICU patients suffer from Intensive Care Unit-Acquired Weakness (ICU-AW). The negative impact of ICU-AW is the prolonged duration of mechanical ventilation. An excessive loss of muscle mass and strength results in physical impairment, frailty, disability, and dependence on others' help. The essential preventive measures include early physiotherapy, tight glucose control, and vitamin D supplementation [5].

Early enteral nutrition is recommended in critically ill patients. The optimal method of administering enteral nutrition remains unknown. The most common practice is continuous enteral feeding; however, this method has recently been called into question in favor of intermittent enteral nutrition administration, where boluses are given 5 to 6 times a day. Optimal muscle protein synthesis requires a pulsatile increase in branch-chain amino acids blood level. In addition, the gastrointestinal tract is an essential endocrine organ producing several important regulatory peptides with hormonal activity. The hormones regulate gastrointestinal motility, gall bladder contraction, pancreatic function, and facilitate insulin secretion, which is important for better control of glycemia [6].

\section{Mental impairment}

The last factor is mental impairment. It includes mental depression, anxiety, and Post-Traumatic Stress Disorder (PTSD). The incidence of the impairment is up to $70 \%$ of patients. Preexisting depression is considered a risk factor. Depression also decreases oral nutrition intake and contributes to the development of malnutrition. A family member of patients can also develop anxiety, depression, and PTSD. The introduction of ICU-diaries seems to be a good helper in prevention of PTSD. The diary is a daily record of a patient's ICU stay written by the healthcare staff or family. Diary can help with the memory gap [3].

\section{Prevention of PICS}

The extension of the $\mathrm{ABCDE}$ bundle reducing the risk of sedation, delirium, and immobility by FGH measures helps prevent the development of all cognitive, physical, and mental disorders.

a) Assess and manage pain

b) Breathing trials and spontaneous breathing trials (sedation protocols must include the routine use of sedation scoring, daily sedation targets, and sedation holidays)

c) Choice of sedation. Benzodiazepines should not be used as
GABA agonists are implicated in the development of delirium. Dexmedetomidine, as a highly selective $\alpha-2$ agonist, is effective in short-term sedation in ICU has opiate sparing properties and is usually well-tolerated.

d) Delirium assessment, daily monitoring (CAM-ICU, environmental management such as a noise reduction, ensuring the circadian light and dark rhythm by melatonin)

e) Early mobility (physiotherapy and nutrition)

f) Family engagement (ICU diary is written for the patient by family member or staff, can help to indicate the orientation of the patient)

g) Good handoff communication (to strengthen the connection between the patient and family members and medical staff)

h) Handout materials on PICS [3,7]

\section{Conclusion}

The mortality of ICU patients decreased. Still, many patients discharged from ICU suffer from PICS with a profound impact on the quality of life and other family members' adverse effects. PICS is a new task for intensive care medicine, highlighting the need to improve critically ill patients' long-term prognosis.

\section{References}

1. Tay MZ, Poh CM, Rénia L, Ary PA, Lisa FP, (2020) The trinity of COVID-19: immunity, inflammation, and intervention. Nature Rev Imunol 28: 1-12.

2. Bektas A, Schurman SH, Franceschi C, Ferrucci L, (2020) A public health perspective of aging: do hyper-inflammatory syndromes such as COVID-19, SARS, ARDS, cytokine storm syndrome, and post-ICU syndrome accelerate short-and long-term inlfammaging? Immun Ageing 17: 23.

3. Inoue S, Hatakeyama J, Kondo Y, Toru H, Hideaki S, et al. (2019) Postintensive cere syndrome: its pathophysiology, prevention, and future directions. Acute Med Surg 6(3): 233-246.

4. Girard TD, Pandharipande P, Ely EW, (2008) Delirium in the intensive care unit. Crit Care 12(3): 3.

5. Zorovic RD, (2016) ICU-acquired weakness. A rehabilitation perspective of diagnosis, treatment, and functional management. CHEST journal 150(4): 966-971.

6. Patel JJ, Rosenthal MD, Heyland DK, (2018) Intermittent versus continuous feeding in critically ill adults. Curr Opin Clin Nutr Metab Care 21(2): 116-120.

7. Colbenson GA, Johnson A, Wilson ME, (2019) Post-intensive care syndrome: impact, prevention, and management. Breathe 15(2): 98101. 